\title{
First Principles Study of Electronic and Magnetic Properties of Co-Doped Armchair Graphene Nanoribbons
}

\author{
Biao Li, ${ }^{1}$ Dahai Xu, ${ }^{1}$ Jun Zhao, ${ }^{1,2}$ and Hui Zeng ${ }^{1,3}$ \\ ${ }^{1}$ School of Physics and Optoelectronic Technology, Yangtze University, Jingzhou, Hubei 434023, China \\ ${ }^{2}$ School of Chemistry and Chemical Engineering, Key Laboratory of Mesoscopic Chemistry of MOE, Nanjing University, \\ Nanjing, Jiangsu 210093, China \\ ${ }^{3}$ School of Physics, Nanjing University, Nanjing, Jiangsu 210093, China
}

Correspondence should be addressed to Jun Zhao; zhaojun@yangtzeu.edu.cn

Received 27 March 2015; Accepted 3 June 2015

Academic Editor: Xuping Sun

Copyright (C) 2015 Biao Li et al. This is an open access article distributed under the Creative Commons Attribution License, which permits unrestricted use, distribution, and reproduction in any medium, provided the original work is properly cited.

Using the first principles calculations, we have studied the atomic and electronic structures of single Co atom incorporated with divacancy in armchair graphene nanoribbon (AGNR). Our calculated results show that the Co atom embedded in AGNR gives rise to significant impacts on the band structures and the FM spin configuration is the ground state. The presence of the Co doping could introduce magnetic properties. The calculated results revealed the arising of spin gapless semiconductor characteristics with doping near the edge in both ferromagnetic (FM) and antiferromagnetic (AFM) magnetic configurations, suggesting the robustness for potential application of spintronics. Moreover, the electronic structures of the Co-doped AGNRs are strongly dependent on the doping sites and the edge configurations.

\section{Introduction}

Graphene is a hexagonal lattice which consists of single atomic layer of $\mathrm{sp}^{2}$ hybridized carbon atoms. Owing to its novel properties arising from the truly two-dimensional nanostructure, graphene has attracted extensive theoretical and experimental investigations since its fabrication in 2004 [1]. The unique electronic and transport properties make the graphene nanomaterial the most promising candidate to be served as the next generation nanodevices to replace siliconbased devices [2]. However, its gapless band structure is a major obstacle for the practical application of graphene in the semiconductor area. As a result, considerable efforts are devoted to opening a moderate band gap for the graphenebased nanomaterials, and many approaches have been proposed in the last decade. More specifically, it has been shown that graphene can be lithographically patterned into quasi one-dimensional graphene nanoribbons (GNRs), and the gap opening can be achieved by the quantum confinement effect in GNRs. The atomic configurations at graphene edge can be characterized by transmission electron microscopy (TEM) and scanning tunneling microscopy (STM) [3]. According to the morphology of graphene edges, the GNRs are conventionally classified into two kinds, namely, armchair edge and zigzag edge [4]. Previous reports have demonstrated that the electronic properties of the GNRs are fundamentally dominated by the atomic arrangement pattern of their edges $[5,6]$.

Application of GNRs for spintronic devices has been limited by their nonmagnetic (NM) characteristics [7]. The introducing of magnetic properties has become one of the most important issues to be investigated. The theoretical research reported previously has shown that the achievement of magnetic properties in graphene nanomaterial can be realized by means of transition metal (TM) atom doping [8]. Correspondingly, experimental fabrication of $\mathrm{Fe}[9,10]$ and Co $[11,12]$ in graphene nanomaterials and the characterization of their magnetic properties have been implemented. In general, there are two routes to introduce TM in graphene and GNRs: adsorption of the TM atom on graphene sheets directly [13-18] and embedment of the TM atom in vacancy defects [19-22]. Although the adsorption of TM atoms on 


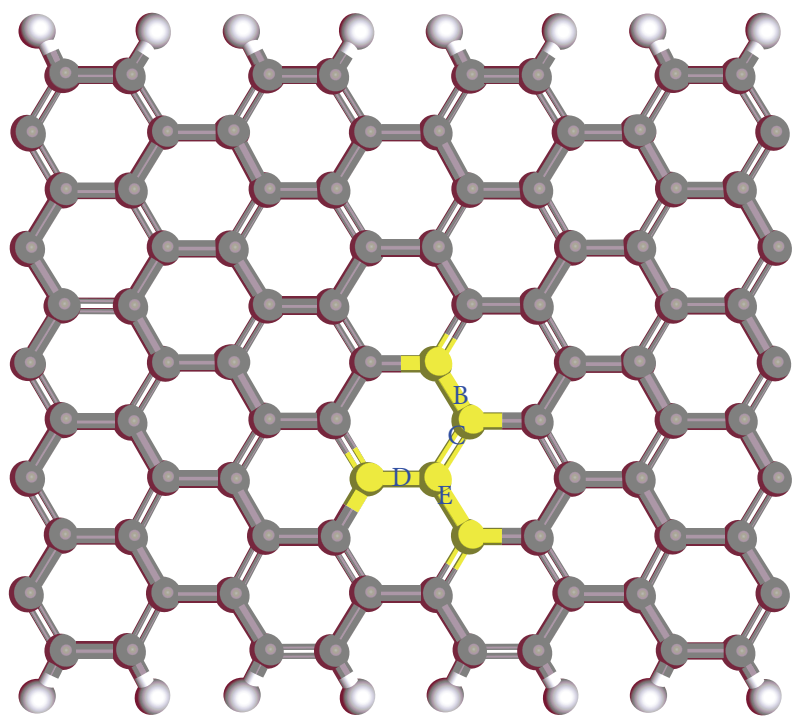

(a)

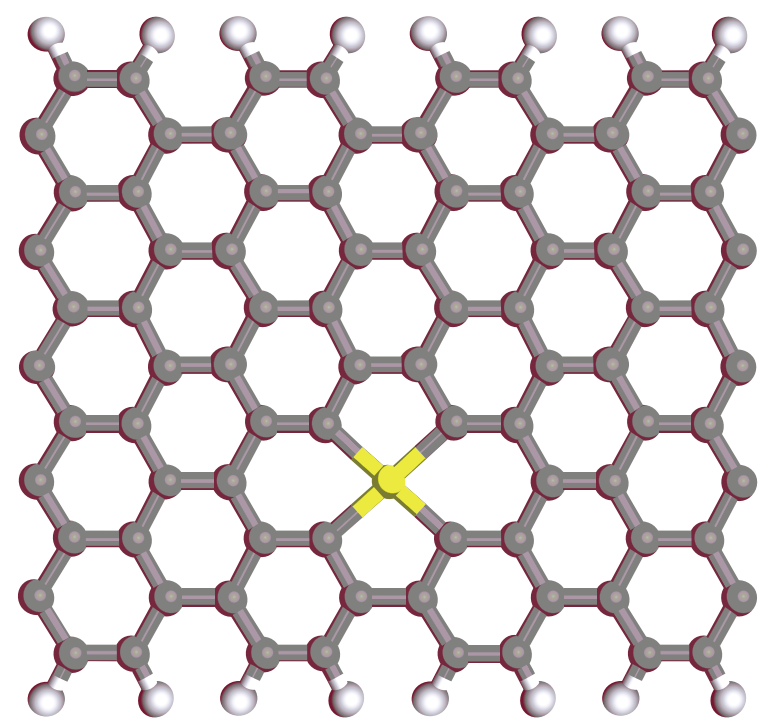

(b)

FIGURE 1: (a) Atomic structures of the 11-AGNR and the Co doping sites in the defective AGNRs. Symbols B to E represent different doping sites under consideration. (b) The ball-and-stick structural model of the Co-D-AGNR. The white, gray, and yellow spheres represent hydrogen, carbon, and cobalt atoms, respectively.

the surface of graphene has large binding energy [17], the doping atom cannot be tightly trapped at room temperature as a result of low migration barrier [19]. Therefore, the migration of the TM in graphene nanostructures limits their application in spintronic devices. In sharp contrast, the TM atoms could incorporate with graphene vacancy, such as single vacancy (SV) [23] and double vacancy (DV) [7]. Hence, TM atoms embedded in vacancy defects have larger binding energy than those adsorbed on graphene surface [19]. Previous studies have shown that TM DV complex has larger magnetic moments than that of the TM-monovacancy composite [19].

Recently, many investigations about TM incorporated with AGNRs have been conducted, such as Mn [24], Fe [25], and $\mathrm{Ni}$ [26]. Previous computational calculations are devoted to the evaluation of electric properties of ZGNRs influenced by the TM doping, such as the doping of Co $[7,21]$ and Au [27]. In this communication, we report an ab initio study of atomic and electronic structure of $\mathrm{Co}$ atoms incorporated in GNR with armchair-shaped edges. The C-C dimer was replaced by Co atoms. We have accessed the influences of Co-DV on the stability and electronic properties associated with various spin configurations of AGNR by using density functional theory calculations. It is found that the presence of the Co doping could introduce magnetic properties and the ferromagnetic coupled spin configuration is the ground state of the Co-doped AGNRs. Moreover, the electronic structures of the Co-doped AGNRs are dependent on the doping sites and the edge configurations.

\section{Method}

The atomic geometry optimizations are implemented by using the density functional theory (DFT) utilized in the code of the Spanish initiative for electronic simulations with thousands of atoms (SIESTA) $[28,29]$. Following the previous convention [5], the width of GNRs with armchair-shaped edges is defined on the basis of the number of dimmer lines $(N)$, for example, $N$-AGNRs. We choose 11 -AGNR consisting of 4 unit cells as supercell to perform computational calculations of electronic and magnetic properties. The standard norm-conserving Troullier-Martins pseudopotential [30] is used to calculate the interaction between valence electrons and the atomic core. The numerical double- $\zeta$ polarized (DZP) basis set and 200 Ry for plane cutoff energy are chosen in the simulation. The generalized gradient approximation (GGA) in the form of Perdew and Burke and Ernzerhof (PBE) is used to calculate exchange correction term [31]. The relaxation of the structures is converged until the force on each atom is less than $0.01 \mathrm{eV} / \AA$.

\section{Results and Discussion}

In order to obtain preferential doping positions of Co atom at room temperature, we have calculated various doping sites at electronic temperature $300 \mathrm{~K}$, which are shown in Figure 1. The carbon atoms at the edge are passivated by hydrogen atoms in the pristine model. The Co atom is embedded in the AGNR nanostructure by replacing two adjacent $\mathrm{C}$ atoms. The different doping sites, from middle to the edge of the AGNRs, are denoted by symbols $B$ to $E$. The doped nanostructures are referred to as Co-B-AGNR, Co-C-AGNR, Co-D-AGNR, and Co-E-AGNR in this content.

In the nonmagnetic (NM) states, the structure model is optimized. The optimized results are shown in Figure 2. Figure 2(a) is the optimized pristine model. All the atoms are found to be in plane. Figure 2(b) is the optimized Co$\mathrm{D}$-AGNR model. It is found that the in-plane structure is 


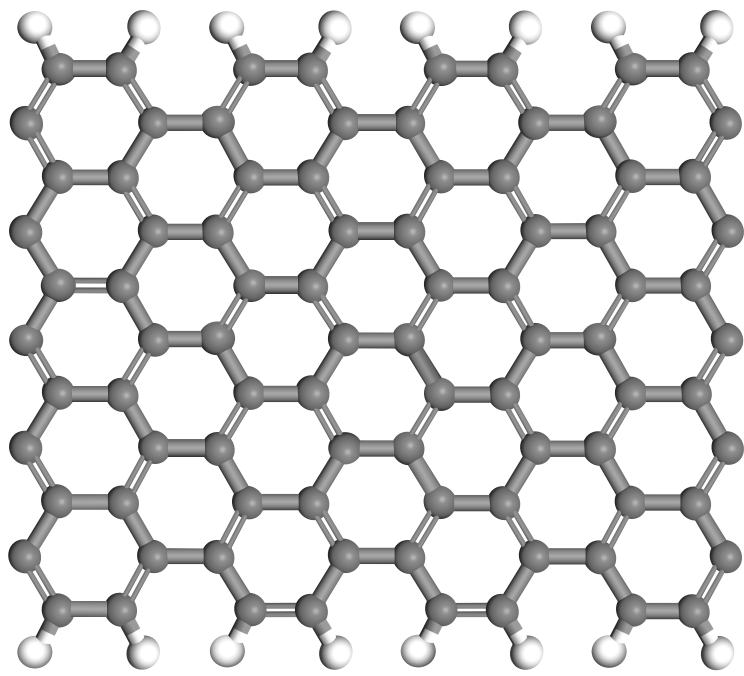

(a)

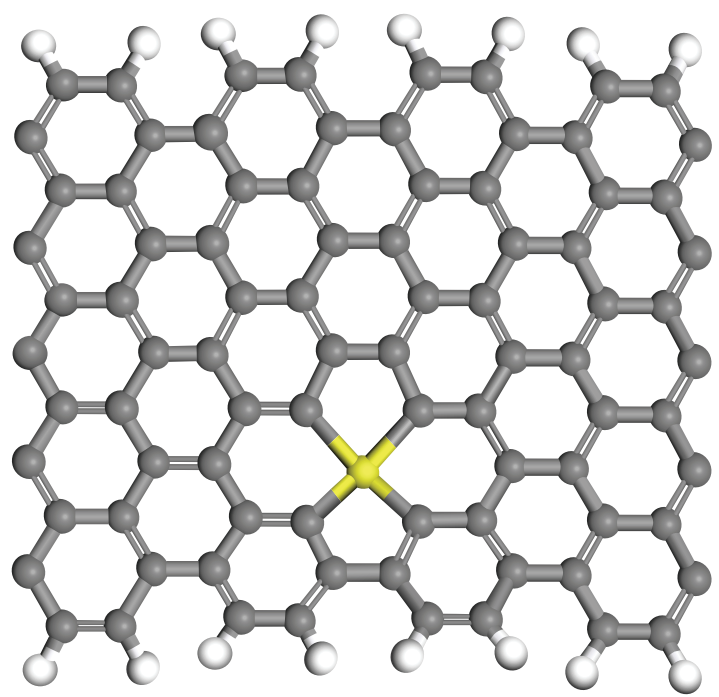

(b)

FIGURE 2: (a) The optimized pristine AGNR nanostructure. (b) The optimized nanostructure of the D site doping configuration.

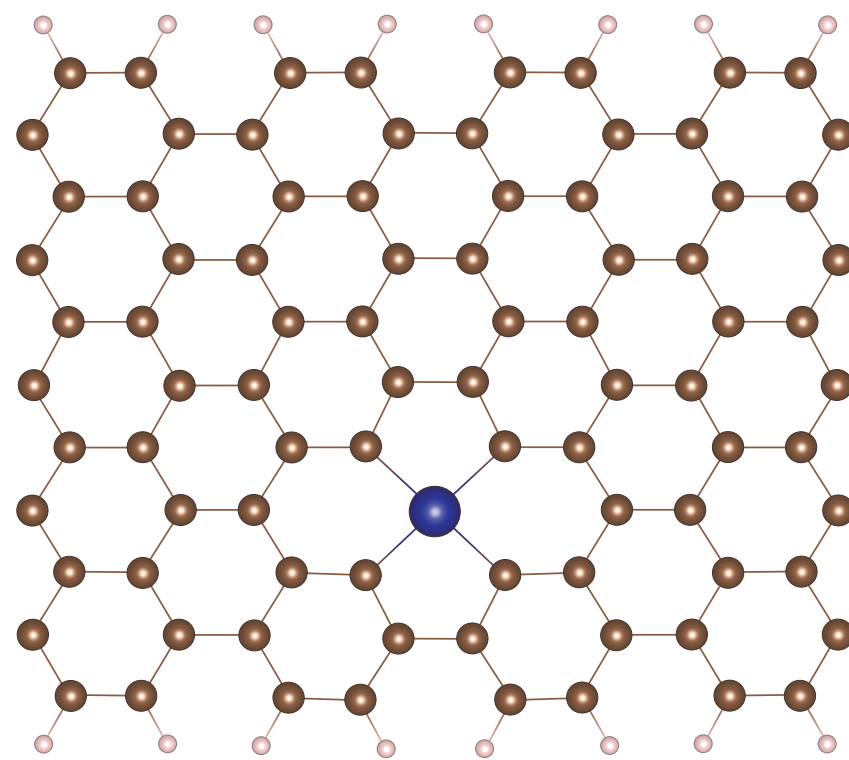

(a)

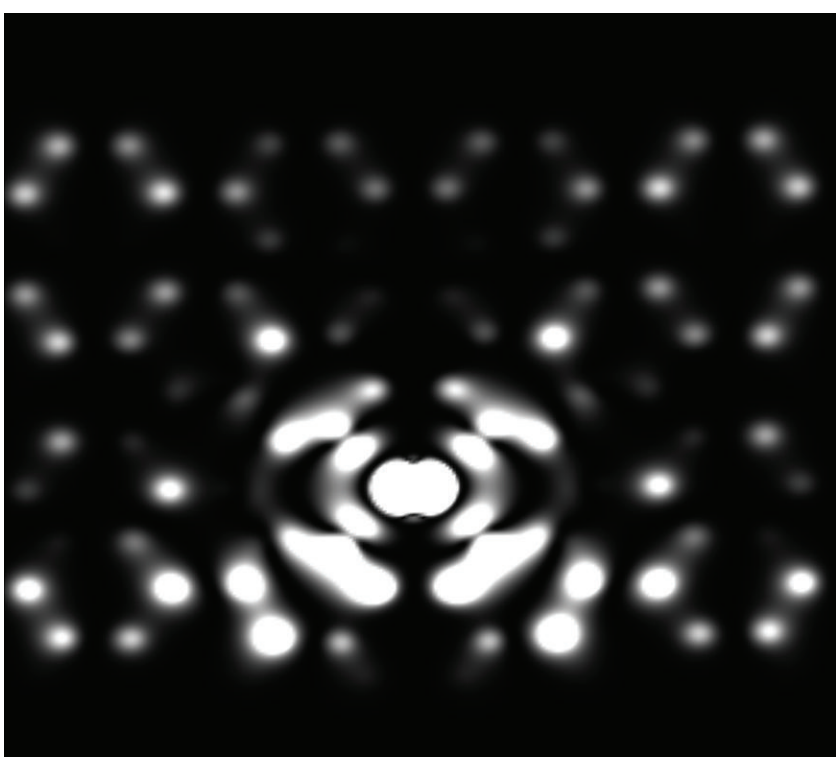

(b)

FIGURE 3: Atomic structures (a) and their simulated STM image (b) of the Co-D-AGNR doping configuration.

destroyed, which is similar to the case in the Au doping in ZGNRs [27]. The carbon atoms on the edge tend to move toward the doping atom. Compared to the adjacent $\mathrm{C}$ atoms, the height of Co atom has been changed after structural reconstruction. With the Co atoms moving from middle to edge, the edge which is close to the Co atom experiences larger distortion than the other side. The curving structure of the Co-doped GNRs is associated with the presence of the Co atom due to its larger mass in comparison to the carbon atom.
In addition, the simulated scanning tunneling microscopy (STM) images are also presented in Figure 3 [32-34], which could be helpful in identifying the Co doping nanostructure in experiments in future.

In order to identify the ground state of the Co-AGNR influenced by different sites, we have also calculated the total energy of the nanostructures with different magnetic configurations, including the antiferromagnetic (AFM) and the ferromagnetic (FM) coupled states. The spin-related 


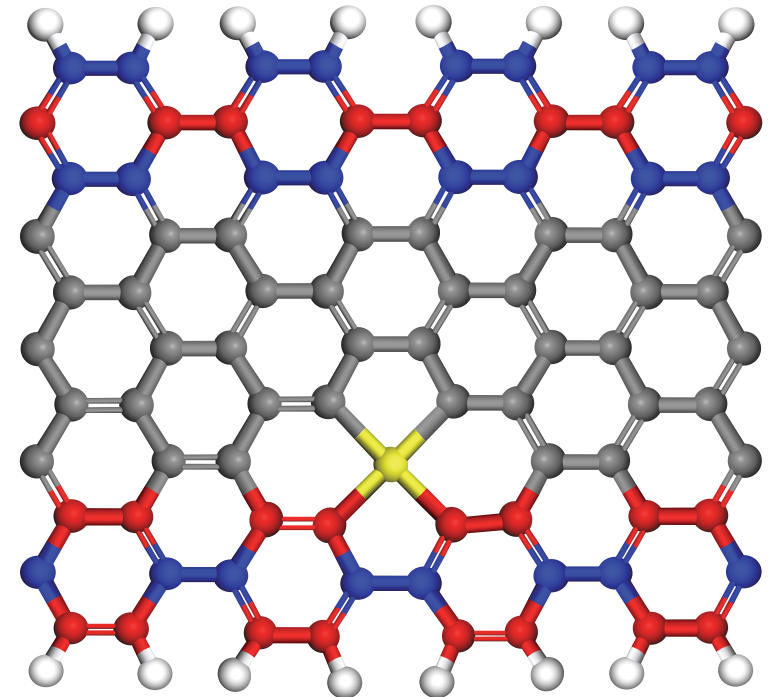

(a)

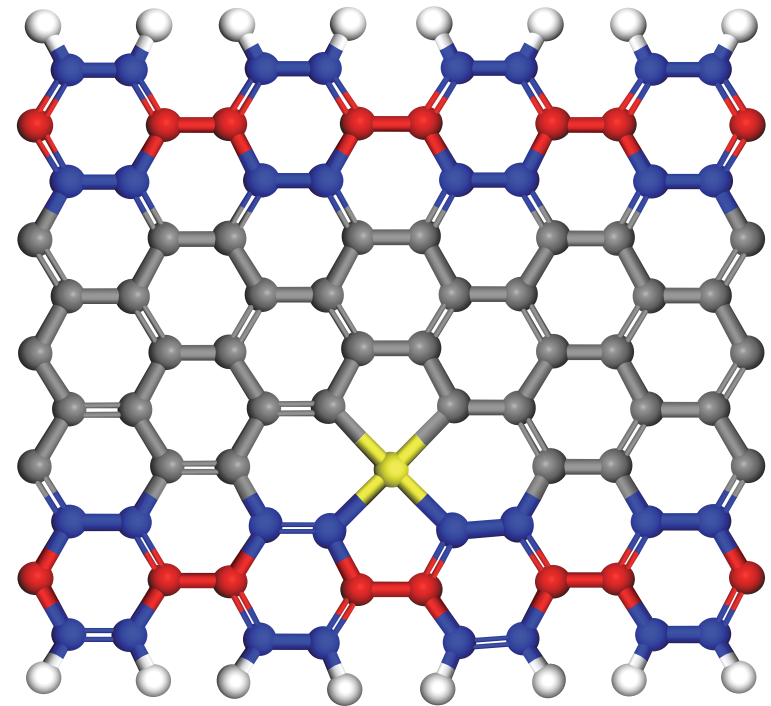

(b)

FIGURE 4: (a) The reconstructed nanostructure of the Co-D-AGNR at AFM state. (b) The reconstructed nanostructure of the Co-D-AGNR at FM state. The blue and red colors denote opposite spin states.

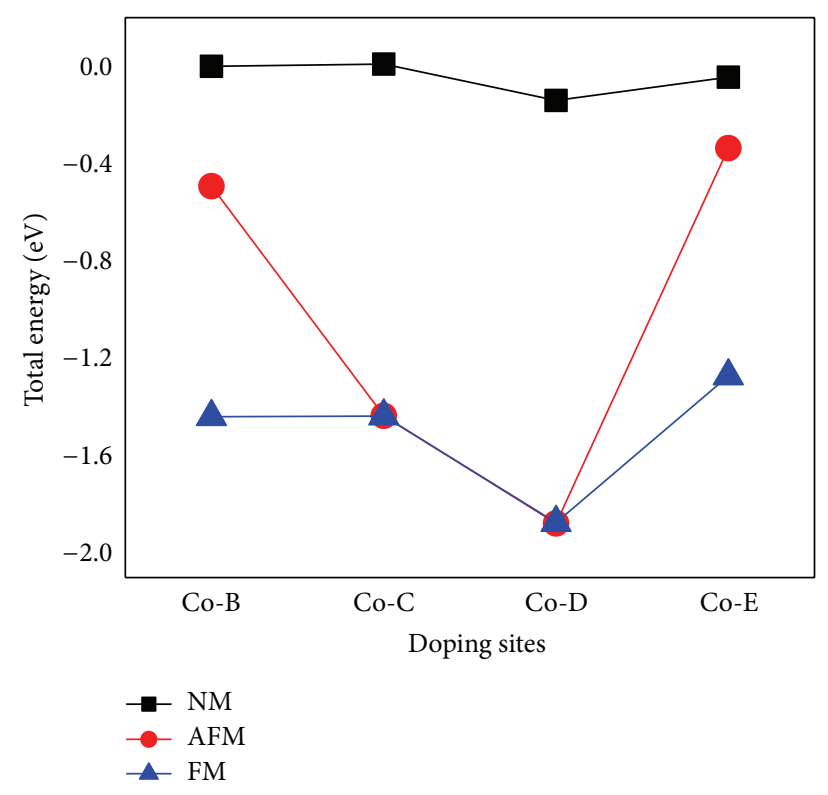

Figure 5: The total energy of different Co-doped AGNRs nanostructures at different spin configurations.

magnetic configurations are shown in Figure 4, and the nanostructures imposed to AFM and FM states are fully relaxed. The calculated results indicate that all atoms are basically in plane under AFM and FM states. The longest Co-C bond length is $1.93 \AA$, which is presented in the Co$\mathrm{D}$-AGNR configuration, and the lowest $\mathrm{Co}-\mathrm{C}$ bond length is
TABle 1: The total spin magnetic moments of different Co-doped AGNRs nanostructures at different spin configurations.

\begin{tabular}{lcc}
\hline Co position & $\begin{array}{c}\text { Magnetic } \\
\text { state }\end{array}$ & $\begin{array}{c}\text { Magnetic } \\
\text { moment }\left(\mu_{B}\right)\end{array}$ \\
\hline B & AFM & 0 \\
& FM & 1.988 \\
C & AFM & 1.979 \\
& FM & -1.979 \\
D & AFM & 2.852 \\
& FM & 1.976 \\
E & AFM & -1.924 \\
& FM & 1.924 \\
\hline
\end{tabular}

$1.87 \AA$ emerging in the Co-E-AFM configuration. This is in a good agreement with previous calculations carried out in the ZGNRs $[7,21]$. The magnetic moments are shown in Table 1.

The total energies of various doping configurations are shown in Figure 5. To analyze the stability of the various magnetic configurations, we thus have calculated the binding energy (BE) by the following equation:

$$
E_{b}=E(\mathrm{Co}-\mathrm{AGNR})+E(\mathrm{C}-\mathrm{C})-E(\mathrm{Co})-E(\mathrm{AGNR}),
$$

where $E$ (Co-AGNR) is the total energy of Co-AGNR, $E(\mathrm{AGNR})$ is the total energy of the pristine AGNR, $E(\mathrm{C}-\mathrm{C})$ is the total energy of $\mathrm{C}$-C dimer in graphene, and $E(\mathrm{Co})$ is the total energy of an isolated Co atom. The $\mathrm{BE}$ is summarized in 


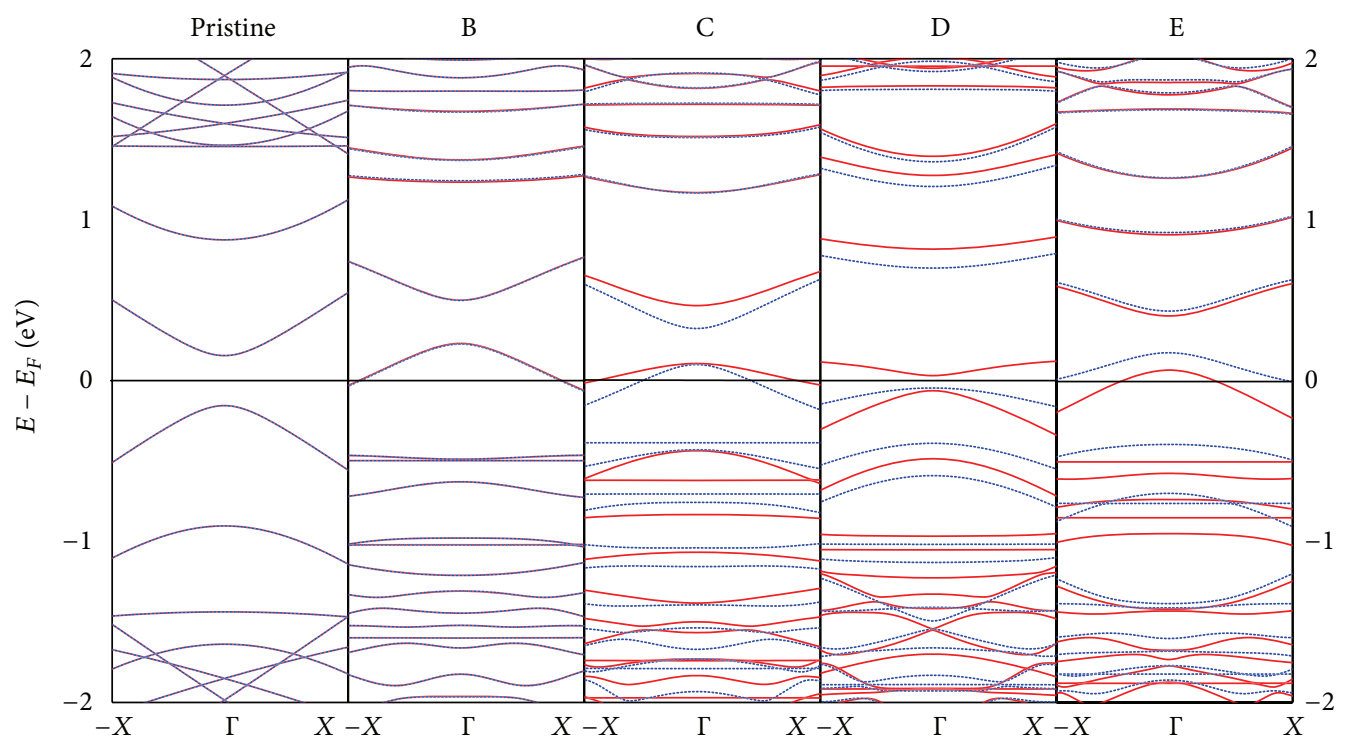

(a)

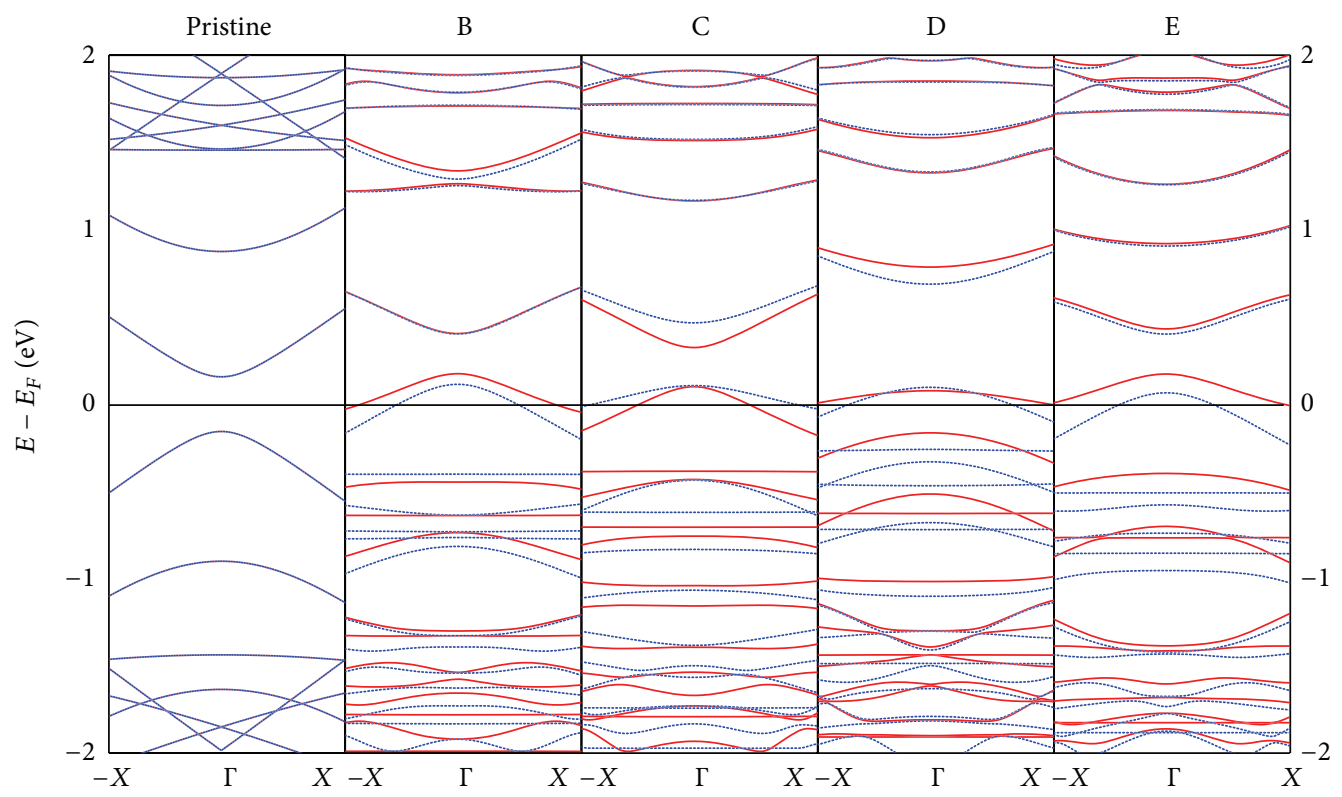

(b)

FIGURE 6: Electronic band structures of Co embedded in AGNRs at different doping sites. (a) and (b) are the spin-polarized results associated with AFM and FM magnetic configurations, respectively. The solid red (dotted blue) line corresponds to spin-up (spin-down) states. The Fermi level is set to zero.

Table 2. Clearly, the Co-D-AGNR configuration has the lowest total energy and the lowest binding energy. Therefore, D site is the most energetically favorable doping configuration. For the $\mathrm{D}$ and $\mathrm{E}$ doping sites, it is noted that the total energy is almost equal under FM and AFM states. Compared with NM and AFM spin configurations, the total energy in the FM state is the lowest one. The FM state configuration is found to be their ground state of these nanostructures.
The spin-polarized band structures of Co-AGNR in AFM state are exhibited in Figure 6(a). Pristine AGNR is fully degenerate with a band gap of $0.31 \mathrm{eV}$, which is in excellent agreement with previous study [35]. The band structure of the Co-B-AGNR is degenerate in sharp contrast to the splitting band structures of $\mathrm{C}-\mathrm{E}$ site doping configuration. The presence of the TM atom in the nanostructure is generally favorable for electron transport by narrowing the electronic 
TABLE 2: The binding energy of different Co-doped AGNRs nanostructures at different spin configurations.

\begin{tabular}{lcc}
\hline Co position & $\begin{array}{c}\text { Magnetic } \\
\text { states }\end{array}$ & $\begin{array}{c}\text { Binding energy } \\
(\mathrm{eV})\end{array}$ \\
\hline B & AFM & 2.438 \\
& FM & 1.482 \\
C & AFM & 1.495 \\
& FM & 1.484 \\
D & AFM & 1.053 \\
& FM & 1.047 \\
E & AFM & 2.594 \\
& FM & 1.649 \\
\hline
\end{tabular}

band gap. For instance, the gap between valence band maximum and conduction band minimum is reduced to $0.08 \mathrm{eV}$ for the Co-D-AGNR configuration. Moreover, the band gap of the doped AGNRs can even be closed, for example, B and $\mathrm{C}$ and $\mathrm{E}$ site doping. It also suggests that the electronic structures of the doped AGNRs significantly depend on the doping sites. Interestingly, E doping configuration can lead to the transition of normal semiconductors to spin gapless semiconductor [36], featuring by gapless electronic structure for one spin channel and gapped electronic structure for opposite spin channel. Therefore, our calculations indicate that doping TM atom near the edge of AGNRs in device fabrication may be beneficial to the real application of spintronics.

The spin-polarized electronic band structures of CoAGNR in FM coupled state are shown in Figure 6(b). Similar to the AFM coupled state, the electronic structures of pristine AGNR in FM state are also degenerate and a band gap of $0.31 \mathrm{eV}$ is observed. All band structures are splitting owing to the presence of Co atom embedded in AGNR, and the Fermi level is found to be substantially downward shifted compared to the pristine case, which resembles the electronic structures under AFM state. For the Co-B-AGNR, the gap between the valence band maximum and conduction band minimum is 0.23 and $0.29 \mathrm{eV}$ for spin-up and spin-down states, respectively. The band structures of Co-C-AGNR and Co-E-AGNR are similar to their corresponding AFM state. The calculated results revealed that the Co-D-AGNR configuration is a spin gapless semiconductor material in the FM state, while it is a fully gapped material in the AFM state. As a result, the electronic transport properties of this nanostructure can be modulated via tuning the magnetic states. On the other hand, the Co-E-AGNR keeps spin gapless semiconductor characteristics in both FM and AFM state, exhibiting the robustness for potential application of spintronics. Overall, our results show that doping site and edge configuration have influences on the electronic structure of Co-doped AGNRs.

The calculated total and projected density of states (TDOS and PDOS, resp.) of these doped nanostructures are shown in Figure 7. In accordance with the electronic band structures described above, the PDOS and the TDOS of the Co-BAGNR are degenerate for opposite spin states. There occurs a peak at $-1 \mathrm{eV}$ both in the TDOS and PDOS, which is consistent with the presence of flat band at this location in the electronic band structure, as shown in Figure 6(a). Here, the localized states in this vicinity thus can be attributed to the Co atom and its contributions to the opposite spin states are almost equivalent. For the Co-C-AGNR under AFM state, the opposite spin states at $-0.38 \mathrm{eV}$ are observed to have distinct differences. And the localized state in the electronic band structure can be recognized to the spin-down state influenced by the Co doping. Hence, for the Co-E-AGNR under AFM state, the presence of the TM doping gives rise to the two flat bands at $-0.5 \mathrm{eV}$ and $-0.9 \mathrm{eV}$ in the spinup band structure. For the FM magnetic configurations, the PDOS are splitting for the Co-B-AGNR nanostructure, which is consistent with the nondegenerate bands around the Fermi level. For the $\mathrm{C}, \mathrm{D}$, and $\mathrm{E}$ doping sites, it is interesting to find that the opposite states in PDOS are flipped. In brief, our calculations of TDOS and PDOS indicate that the Co atom could have pronounced influences on the electronic structures around the Fermi level that are favorable for electronic transport, which are dependent on the doping sites.

\section{Conclusions}

We have performed computational calculations to study the influences of single Co atom doping on the electronic structures of the 11-AGNRs. The FM spin configuration is the ground state for all doped sites. Our calculated results revealed that the Co-D-AGNR is the most stable doping configuration, and the electronic transport properties of this nanostructure can be tuned between spin gapless semiconductor and gapped nanomaterial by changing magnetic states. In addition, the Co-E-AGNR keeps spin gapless semiconductor characteristics in both FM and AFM states, exhibiting the robustness for potential application of spintronics. The embedding of Co atoms in the AGNRs leads to the downward shift of Fermi level and gives rise to significant impacts on their electronic band structures. The electronic structures of Co-doped AGNRs are strongly dependent on the doping sites and the edge configurations.

\section{Conflict of Interests}

The authors declare that there is no conflict of interests regarding the publication of this paper.

\section{Acknowledgments}

The authors are grateful to Dr. J.-W. Wei for helpful discussions, Professor Ma for assistance on calculations in the High Performance Computing Center of Nanjing University, and Dr. Y.-H. Zhao for performing partial simulations in HPCC@SICNU. This work is financially supported by the Natural Science Foundation of China (Grant nos. 11304022, 


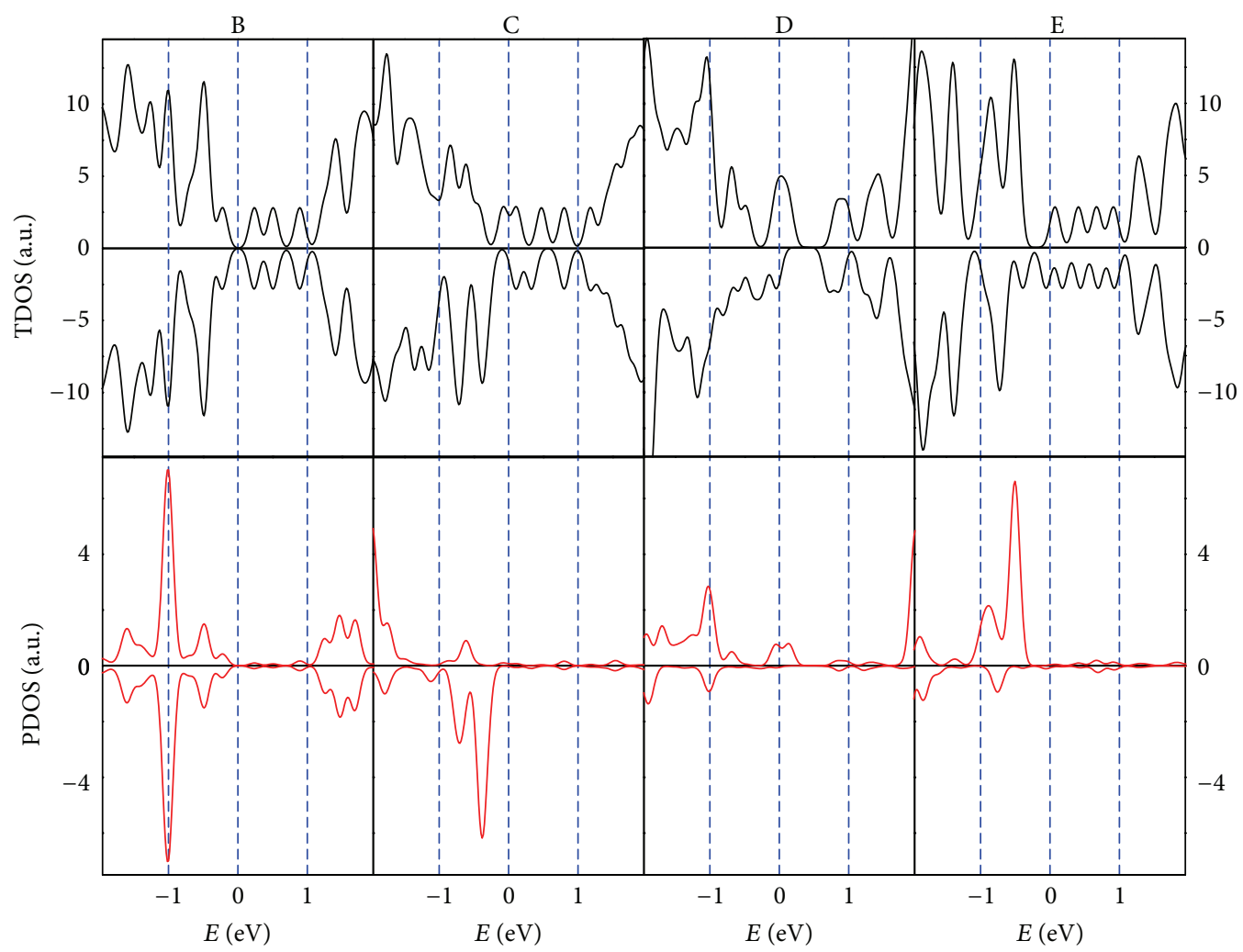

(a)

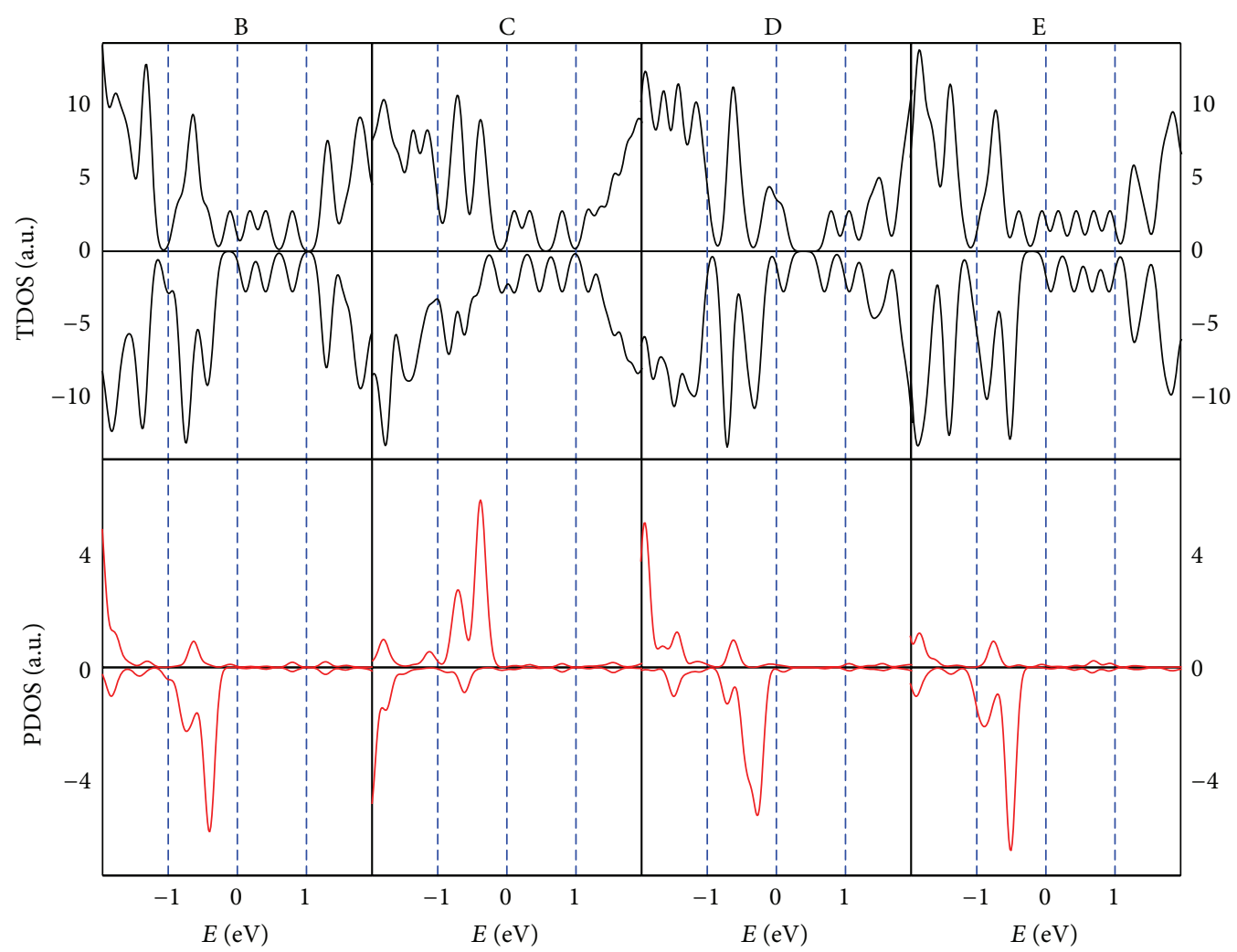

(b)

Figure 7: Total (black line) and projected (red line) density of states of the Co-B-AGNR, Co-C-AGNR, Co-D-AGNR, and Co-E-AGNR under different magnetic configurations. (a) and (b) are the spin-polarized results associated with AFM and FM magnetic configurations, respectively. Spin-up and spin-down states are denoted by positive and negative values, respectively. The Fermi level is set to zero. 
11347010, 11404037, and 11204391), China Postdoctoral Science Foundation (Grant no. 2015M571729), and the Scientific and Technological Research Program of Chongqing Municipal Education Commission (Grant no. KJ130831).

\section{References}

[1] K. S. Novoselov, A. K. Geim, S. V. Morozov et al., "Electric field in atomically thin carbon films," Science, vol. 306, no. 5696, pp. 666-669, 2004.

[2] Y. Zhang, Y.-W. Tan, H. L. Stormer, and P. Kim, "Experimental observation of the quantum Hall effect and Berry's phase in graphene," Nature, vol. 438, no. 7065, pp. 201-204, 2005.

[3] A. K. Geim and K. S. Novoselov, "The rise of graphene," Nature Materials, vol. 6, no. 3, pp. 183-191, 2007.

[4] K. Nakada, M. Fujita, G. Dresselhaus, and M. S. Dresselhaus, "Edge state in graphene ribbons: nanometer size effect and edge shape dependence," Physical Review B, vol. 54, no. 24, pp. 1795417961, 1996.

[5] A. H. C. Neto, F. Guinea, N. M. R. Peres, K. S. Novoselov, and A. K. Geim, "The electronic properties of graphene," Reviews of Modern Physics, vol. 81, no. 1, pp. 109-162, 2009.

[6] H. Terrones, R. Lv, M. Terrones, and M. S. Dresselhaus, "The role of defects and doping in $2 \mathrm{D}$ graphene sheets and $1 \mathrm{D}$ nanoribbons," Reports on Progress in Physics, vol. 75, no. 6, Article ID 062501, 2012.

[7] A. T. Lee and K. J. Chang, "Effect of edges on the stability and magnetic interaction of Co atoms embedded in zigzag graphene nanoribbons," Physical Review B, vol. 87, no. 8, Article ID 085435, 2013.

[8] K. Sato, L. Bergqvist, J. Kudrnovský et al., "First-principles theory of dilute magnetic semiconductors," Reviews of Modern Physics, vol. 82, no. 2, pp. 1633-1690, 2010.

[9] A. W. Robertson, B. Montanari, K. He et al., "Dynamics of single fe atoms in graphene vacancies," Nano Letters, vol. 13, no. 4, pp. 1468-1475, 2013.

[10] Z.-Y. He, K. He, A. W. Robertson et al., "Atomic structure and dynamics of metal dopant pairs in graphene," Nano Letters, vol. 14, no. 7, pp. 3766-3772, 2014.

[11] H.-T. Wang, Q.-X. Wang, Y.-C. Cheng et al., "Doping monolayer graphene with single atom substitutions," Nano Letters, vol. 12, no. 1, pp. 141-144, 2012.

[12] F. Donati, L. Gragnaniello, A. Cavallin et al., "Tailoring the magnetism of Co atoms on graphene through substrate hybridization," Physical Review Letters, vol. 113, no. 17, Article ID 177201, 2014.

[13] K. T. Chan, J. B. Neaton, and M. L. Cohen, "First-principles study of metal adatom adsorption on graphene," Physical Review $B$, vol. 77, no. 23, Article ID 235430, 2008.

[14] H. Sevinçli, M. Topsakal, E. Durgun, and S. Ciraci, "Electronic and magnetic properties of $3 \mathrm{~d}$ transition-metal atom adsorbed graphene and graphene nanoribbons," Physical Review B, vol. 77, no. 19, Article ID 195434, 2008.

[15] Y. Virgus, W. Purwanto, H. Krakauer, and S.-W. Zhang, "Stability, energetics, and magnetic states of cobalt adatoms on graphene," Physical Review Letters, vol. 113, no. 17, Article ID 175502, 2014.

[16] V. A. Rigo, T. B. Martins, A. J. R. Da Silva, A. Fazzio, and R. H. Miwa, "Electronic, structural, and transport properties of Nidoped graphene nanoribbons," Physical Review B, vol. 79, no. 7, Article ID 075435, 2009.
[17] K. T. Chan, H. Lee, and M. L. Cohen, "Gated adatoms on graphene studied with first-principles calculations," Physical Review B-Condensed Matter and Materials Physics, vol. 83, no. 3, Article ID 035405, 2011.

[18] S. R. Power, V. M. de Menezes, S. B. Fagan, and M. S. Ferreira, "Magnetization profile for impurities in graphene nanoribbons," Physical Review B, vol. 84, no. 19, Article ID 195431, 2011.

[19] A. V. Krasheninnikov, P. O. Lehtinen, A. S. Foster, P. Pyykkö, and R. M. Nieminen, "Embedding transition-metal atoms in graphene: structure, bonding, and magnetism," Physical Review Letters, vol. 102, no. 12, Article ID 126807, 2009.

[20] E. J. G. Santos, D. Sánchez-Portal, and A. Ayuela, "Magnetism of substitutional Co impurities in graphene: realization of single $\pi$ vacancies," Physical Review B, vol. 81, no. 12, Article ID 125433, 2010.

[21] S. Lisenkov, A. N. Andriotis, and M. Menon, "Magnetic anisotropy and engineering of magnetic behavior of the edges in Co embedded graphene nanoribbons," Physical Review Letters, vol. 108, no. 18, Article ID 187208, 2012.

[22] D. W. Boukhvalov and M. I. Katsnelson, "Destruction of graphene by metal adatoms," Applied Physics Letters, vol. 95, no. 2, Article ID 023109, 2009.

[23] N. K. Jaiswal and P. Srivastava, "Ab-initio study of Co-doped Zigzag graphene nanoribbons," AIP Conference Proceedings, vol. 144, no. 1, pp. 805-806, 2012.

[24] S. S. Chauhan, P. Srivastava, and A. K. Shrivastava, "Band gap engineering in doped graphene nanoribbons: an ab initio approach," Solid State Communications, vol. 154, no. 1, pp. 6971, 2013.

[25] N. K. Jaiswal and P. Srivastava, "Fe-doped armchair graphene nanoribbons for spintronic/interconnect applications," IEEE Transactions on Nanotechnology, vol. 12, no. 5, pp. 685-691, 2013.

[26] N. K. Jaiswal and P. Srivastava, "Structural stability and electronic properties of Ni-doped armchair graphene nanoribbons," Solid State Communications, vol. 151, no. 20, pp. 1490-1495, 2011.

[27] X.-H. Hu, W. Zhang, L.-T. Sun, and A. V. Krasheninnikov, "Gold-embedded zigzag graphene nanoribbons as spin gapless semiconductors," Physical Review B, vol. 86, no. 19, Article ID 195418, 2012.

[28] P. Ordejón, E. Artacho, and J. M. Soler, "Self-consistent order-N density-functional calculations for very large systems," Physical Review B, vol. 53, no. 16, pp. R10441-R10444, 1996.

[29] J. M. Soler, E. Artacho, J. D. Gale et al., "The SIESTA method for ab initio order- $N$ materials simulation," Journal of Physics: Condensed Matter, vol. 14, no. 11, pp. 2745-2779, 2002.

[30] N. Troullier and J. L. Martins, "Efficient pseudopotentials for plane-wave calculations," Physical Review B, vol. 43, no. 3, pp. 1993-2006, 1991.

[31] J. P. Perdew, K. Burke, and M. Ernzerhof, "Generalized gradient approximation made simple," Physical Review Letters, vol. 77, no. 18, pp. 3865-3868, 1996.

[32] G. Kresse and J. Furthmüller, "Efficient iterative schemes for ab initio total-energy calculations using a plane-wave basis set," Physical Review B-Condensed Matter and Materials Physics, vol. 54, no. 16, pp. 11169-11186, 1996.

[33] G. Kresse and J. Furthmüller, "Efficiency of ab-initio total energy calculations for metals and semiconductors using a plane-wave basis set," Computational Materials Science, vol. 6, no. 1, pp. 15-50, 1996. 
[34] K. Momma and F. Izumi, "VESTA 3 for three-dimensional visualization of crystal, volumetric and morphology data," Journal of Applied Crystallography, vol. 44, no. 6, pp. 1272-1276, 2011.

[35] F. Ma, Z.-K. Guo, K.-W. Xu, and P. K. Chu, "First-principle study of energy band structure of armchair graphene nanoribbons," Solid State Communications, vol. 152, no. 13, pp. 1089-1093, 2012.

[36] X.-L. Wang, "Proposal for a new class of materials: spin gapless semiconductors," Physical Review Letters, vol. 100, no. 15, Article ID $156404,2008$. 

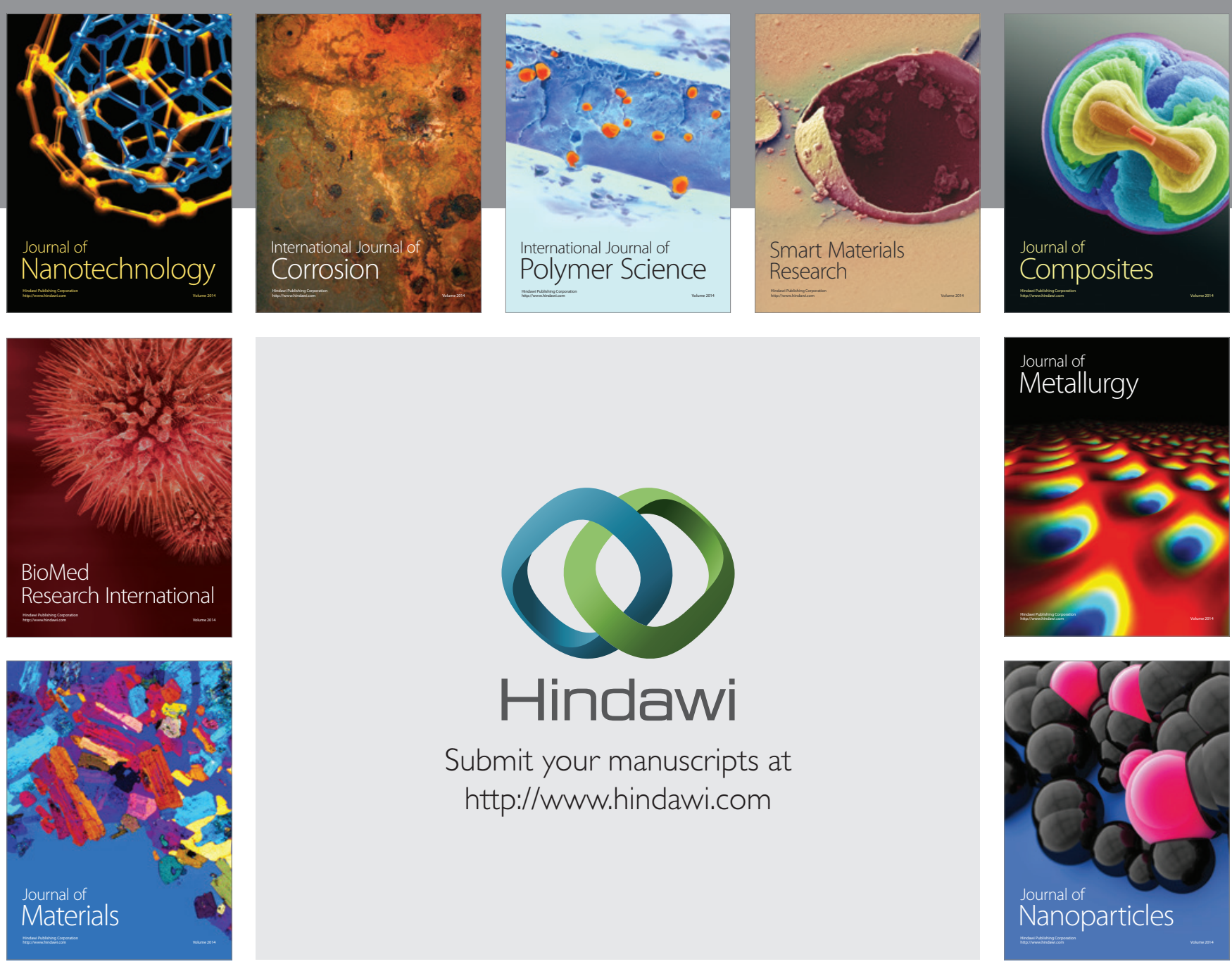

Submit your manuscripts at http://www.hindawi.com
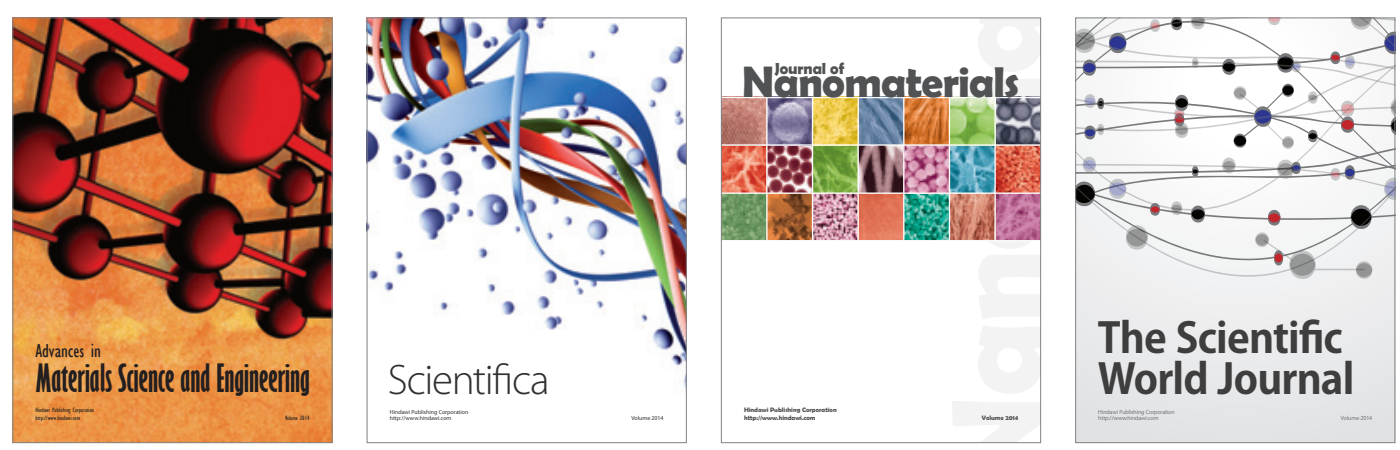

\section{The Scientific World Journal}
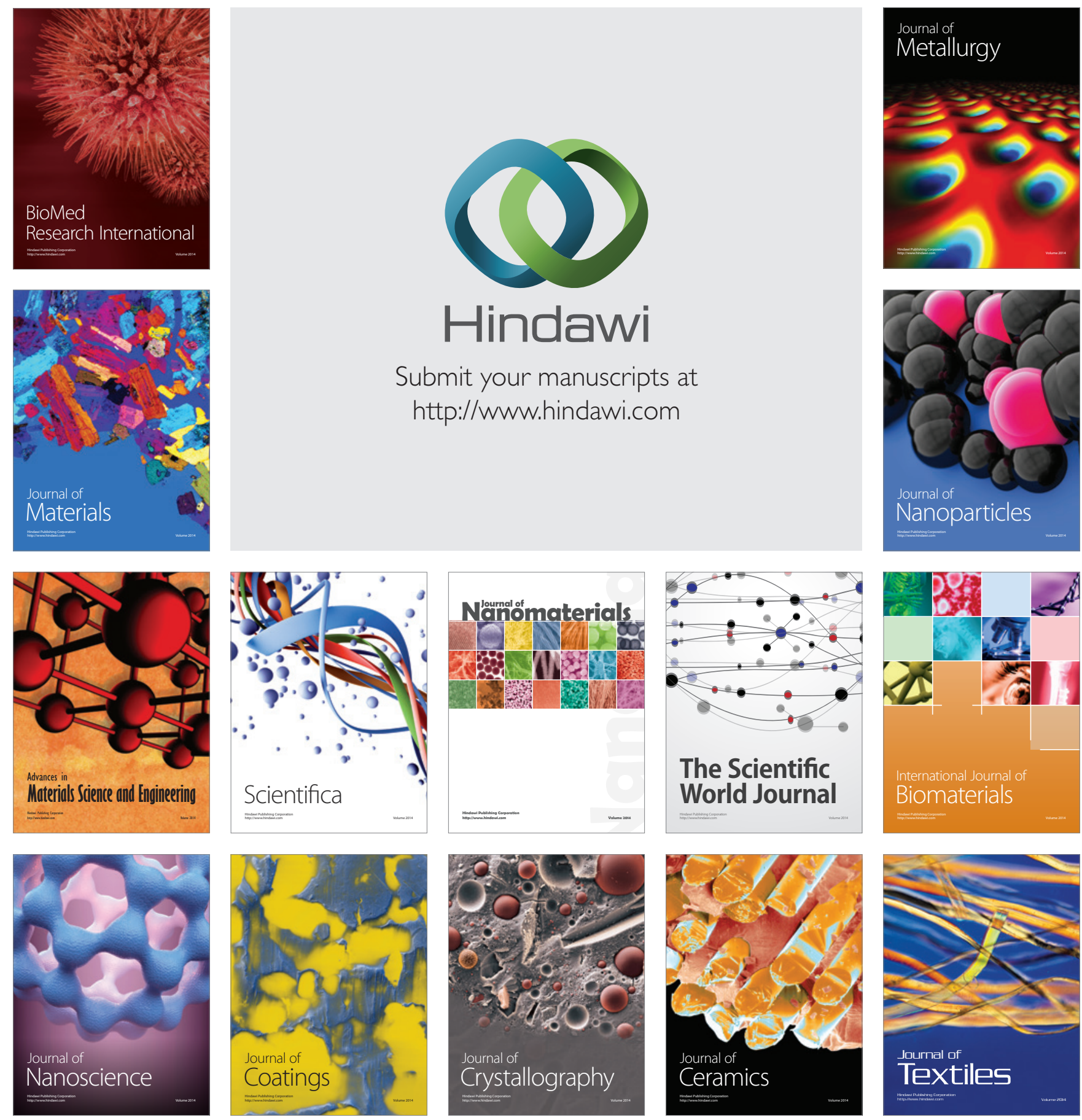